\title{
Sentimientos y cambios en niños y adolescentes con cáncer en Montería (2019)
}

\author{
Feelings and changes in children and adolescents with cancer in Monteria (2019)
}

Sentimentos e mudanças em crianças e adolescentes com câncer em Montería (2019)

\author{
Elsy Cecilia Puello-Alcocer ${ }^{{ }^{*}}$ \\ Katy Karina Herrera-Espitia ${ }^{2}$ \\ Sanyi Carolina German-Orozco ${ }^{3}$
}

\section{Resumen}

Objetivo: Comprender los sentimientos de los niños y adolescentes con diagnóstico de cáncer, a través de la indagación de los cambios que experimentan. Materiales y Métodos: Estudio cualitativo con aproximación fenomenológica Se contó con la participación de 5 niños y adolescentes con diagnóstico de cáncer, atendidos en la ciudad de Montería, Córdoba. Fueron seleccionados mediante la técnica de muestreo bola de nieve. A los participantes se les aplicó una entrevista semiestructurada y observación participante hasta alcanzar el nivel de saturación. Resultados: Se identificaron sentimientos de esperanza y de frustración. Los participantes perciben que sus vidas se transformaron, que su interacción social disminuyó al igual que las actividades educativas. De igual forma sintieron miedo y dolor al tener que enfrentar los estigmas que tiene la sociedad sobre esta enfermedad; no obstante, resaltan que ahora reciben más expresiones de amor y comprensión de toda su familia. Conclusión: Los niños y adolescentes con cáncer expresan que no es fácil afrontar todos los cambios y temores que viven; sin embargo, le otorgan a esta experiencia un significado de transformación y crecimiento personal; de forma paralela, experimentan miedos, dolor, preocupación, temor a la muerte e incertidumbre, además de enfrentarse a las representaciones sociales de esta patología.

Palabras claves: Neoplasias; emociones; pediatría; enfermería.

\section{Abstract}

Objective: Understand the feelings of children and adolescents diagnosed with cancer through research about the changes they experience. Materials and Methods: Qualitative study with a phenomenological approach, based on the participation of 5 children and adolescents diagnosed with cancer and taken care of in Monteria, Cordoba, Colombia, which were selected through the snowball sampling technique. The participants went through a semi-structured interview and observation, until reaching the saturation level. Results: Feelings of hope as well as frustration were identified; the participants perceived that their lives were transformed, their social interaction lowered as well as their educational activities, they felt fear and pain when facing so-

\section{Autor de correspondencia}

${ }^{1 *}$ Enfermera, Magister en Desarrollo Social, Docente Titular Programa de Enfermería, Universidad de Córdoba. Córdoba, Colombia. Correo: epuello@correo.unicordoba. edu.co (iD)

${ }^{2}$ Enfermera. Joven investigador, Universidad de Córdoba. Córdoba, Colombia. Correo: karinaherreespi19@gmail.com (D)

${ }^{3}$ Enfermera. Joven investigador, Universidad de Córdoba. Córdoba, Colombia. Correo: sanyiunicords@gmail.com (iD

(C) Universidad Francisco de Paula Santander. Este es un artículo bajo la licencia CC-BY-NC-ND

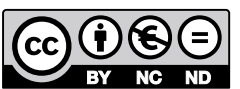


cial stigmas about this disease, now they receive more expressions of love and comprehension from their family. Conclusion: Children and adolescents with cancer express not being easy to face all the changes and fears that cancer creates, however, they give this experience a meaning of personal transformation and growth, at the same time, they also experience fear, pain, preoccupation, fear of death and uncertainty, as well as facing social representations of cancer.

Keywords: Neoplasm; emotions; pediatrics; nursing.

\section{Resumo}

Objetivo: Compreender os sentimentos de crianças e adolescentes com diagnostico de câncer através da indagação das mudanças que experimentam Materiais e Métodos: Estudo qualitativo com aproximação fenomenológica que estudou cinco crianças e adolescentes com diagnostico de câncer atendidos na cidade de Montería, Córdoba, selecionados pelo sistema "bola de neve". Foi aplicada a entrevista semiestruturada e observação participante aos sujeitos estudados até alcançar níveis de saturação. Resultados: Identificaram-se sentimentos de esperança conjuntamente com frustração; os participantes percebem que as suas vidas se transformaram, a sua interação social diminuiu junto com as atividades de ensino, sentiram medo e dor ao enfrentar-se ao preconceito que a sociedade tem dessa doença, entretanto recebem maior compreensão e expressões de amor das suas famílias. Conclusão Global: As crianças e adolescentes com câncer expressam não ser fácil afrontar as mudanças e medos que gera o câncer, entretanto, consideram a experiência transformadora e que acrescenta ao crescimento pessoal, embora experimentam medos, dor, preocupação, temor da morte e incerteza, além disso de enfrentar-se às representações sociais do câncer.

Palavras-chave: Neoplasias; emoções; pediatria; enfermagem.

\section{Introducción}

El cáncer es la segunda causa de mortalidad en el mundo atribuyéndosele 8,8 millones de muertes (1), con la característica que no hace distinción entre grupos poblacionales, porque todas las personas están expuestas de una manera u otra a esta enfermedad. En lo que respecta al cáncer en niños y adolescentes "en Colombia, se presentan 1.322 nuevos casos de cáncer en menores de 18 años" (2), y hasta el 28 de diciembre de 2019, semana epidemiológica 52 (3), se han notificado 1.780 casos de cáncer en menores de 18 años. Estas cifras son preocupantes si se toma en consideración que anualmente (4) se atienden entre 1.500 y 1.600 niños y de estos mueren aproximadamente entre 765 y 816 durante los 5 años del pos diagnóstico.

El imaginario sobre el cáncer varía según el sujeto, pero para la mayoría de las personas este es sinónimo de pérdidas, sufrimiento, dolor y desesperanza, identificándose que dichas representaciones tienen su soporte en las altas cifras de mortalidad por cáncer. Desde esta perspectiva, esta patología es entendida como una "sentencia de muerte" pena o castigo que se equipara con el final de la vida, aspecto que influye para que esta enfermedad sea concebida desde el temor y el ocultamiento (5).

El diagnóstico de un niño con cáncer altera el funcionamiento personal, familiar y comunitario, porque general- mente se producen cambios en la dinámica y en la vida del menor. Factores que requieren el desarrollo de procesos adaptativos, de tal forma que le permitan enfrentar las crisis que genera la enfermedad, particularmente porque durante el proceso, ocurren estancias hospitalarias prolongadas y tratamientos que podrían ocasionar estados de ansiedad, depresión, sentimientos de soledad, cambios físicos e inmunitarios, así como retraso en el desarrollo psicomotor y escolar (6-8).

Por ello, el cuidado de enfermería debe estar orientado hacia la vigilancia y detección de complicaciones, tales como: evitar signos y síntomas físicos propios del tratamiento; intervenir los factores emocionales, psicosociales durante todas las fases de la enfermedad (9); fortalecer las relaciones de interdependencia y el afrontamiento positivo (10); e intervenir en un síntoma específico, como el dolor o la ansiedad en el marco de una consulta temprana por enfermería, para contribuir de forma idónea a la totalidad del cuidado (11).

En los inicios de la enfermedad cuando se diagnostica al paciente, se generan cambios de gran impacto en la vida del individuo. Según Bellver y Verdet (12) el diagnóstico, el tratamiento constante y los sucesivos efectos secundarios de la enfermedad, tales como la pérdida de cabello, náuseas, vómitos, cambios en la piel, diarrea y dolor están correlacionados con fuertes niveles de ten- 
sión y carga psicológica. Razón por la cual, estos fuertes cambios o secuelas que se ven reflejados en el individuo, tienen la capacidad de deteriorar no solo la salud de la persona, sino también el progreso en la construcción de la personalidad y la imagen corporal, que son elementos de importancia en el ciclo vital individual en el que se encuentre cada ser humano.

Los cambios físicos en el proceso oncológico están ligados a los cambios sociales, tales como la sensación de cansancio y vomito que les impide realizar las actividades cotidianas y recreativas que hacían habitualmente; por otro lado, la alopecia podría afectar su autoestima y limitar su vida social. Al respecto Muñiz et al. (13), agregan que los síntomas físicos y la progresión de la enfermedad favorecen la aparición de los problemas en las relaciones sociales, porque para un niño y un adolescente no debe ser fácil experimentar estos cambios en su apariencia y estado de salud, haciéndose necesario reconocer su valentía, dado que no es nada fácil ser niño y mucho menos adolescente, y vivir con cáncer, lo que sin duda es un acto heroico.

En este orden de ideas, los cambios que se generan a partir del diagnóstico de cáncer afectan la calidad de vida del paciente oncológico. Desde esta perspectiva García y Lucio (14), consideran que la calidad de vida de los adolescentes con cáncer, no solo se ve afectada durante el tratamiento, sino también en todos aquellos pacientes que se encontraban en seguimiento y para aquellos que presentaron recaídas. De ahí, que la adaptación y la lucha de los pacientes oncológicos es un proceso constante, hasta después de haber logrado vencer el cáncer fisiológicamente, y no conforme con eso tienen que enfrentarse a las secuelas que este les deja.

Los cambios sociales y los asociados al tratamiento oncológico influyen significativamente en la calidad de vida de los niños y adolescentes con cáncer $(15,16)$. Soportar la caída del cabello, las sensaciones de cansancio, el vómito sumado al aislamiento social, no poder ir a la escuela, la estancia hospitalaria, dejar a un lado los juegos en el parque o en la calle con sus amigos, no ir a cine, no poderse comer un helado en las noches o invitar a la chica que le gusta a comer a su lugar favorito, son algunos de los aspectos más difíciles de la enfermedad. Así se evidencia en el estudio realizado por Llanta et al. (17), en donde se muestra que las experiencias más negativas en este tipo de pacientes, se asocian a los efectos producidos por la quimioterapia y a las preocupaciones sociales, familiares y emocionales.
El proceso oncológico podría asemejarse a una montaña rusa de emociones, porque los sentimientos y en general el sentir de los niños y adolescentes con esta enfermedad es algo único que solo ellos podrían explicar de forma correcta. En este caso, es necesario precisar que tanto el miedo, la frustración, la tristeza, la rabia y la impotencia; así como el optimismo, la ilusión, la valentía, la tranquilidad, son sentimientos que los profesionales de la salud no deben olvidar al momento de realizar sus intervenciones en los pacientes oncológi$\cos$. Factores que se deben tener en cuenta por cuanto se constituyen en nutrientes que facilitan una adecuada adherencia al tratamiento, donde además la adaptación a la enfermedad debe contar con un equipo interdisciplinario que aborde al paciente oncológico de forma global, no solo desde lo físico y lo biológico (18).

Saber y comprender los sentimientos de los niños y adolescentes con cáncer, favorece la oferta de un cuidado humanizado e integral y contribuye para que el binomio paciente-familia afronte y se adapte a la patología y a sus implicaciones de la mejor forma posible.

Con respecto, a los cambios relacionados con el diagnóstico de cáncer, desde la perspectiva de Callista Roy, están inmersos en los modos de adaptación y estimulan la reacción del paciente para dar una respuesta positiva como resultado de un proceso de adaptación o por el contrario una respuesta ineficaz, marcada por la no adaptación a la enfermedad (19). Por tal motivo, los sentimientos que tienen los niños y adolescentes frente al diagnóstico del cáncer y los cambios relacionados con la enfermedad, son diferentes para cada persona.

Según Roy, el profesional de enfermería, debe procurar el uso de sus conocimientos científicos y habilidades adquiridas para: identificar, clasificar, relacionar las características del estímulo, las actitudes, el comportamiento y en general, las conductas que influyen positivamente en el estado de salud del individuo. Por ello se hace necesario, generar estrategias que mejoren la adaptación de las personas, sobretodo en su interacción con el entorno, de tal forma que todos los cambios que se experimentan ante la presencia de un estímulo no esperado, impacte positivamente en el proceso de adaptación (19).

Este tipo de investigaciones, demuestran una vez más el cumplimiento de la misión social y científica de las instituciones de educación superior (20), en la medida en que estos se realizan en colectivos vulnerables, frágiles y resaltan la importancia de escuchar la información que 
brinda el sujeto de cuidado para contribuir a un abordaje integral y humanizado del mismo. En consecuencia, recolectar información sobre los sentimientos que tienen los niños y adolescentes frente al diagnóstico de cáncer, atendidos en la ciudad de Montería, Córdoba (Colombia), es un aspecto de interés, porque permite comprender los sentimientos y los cambios que se presentan en el proceso natural de la enfermedad; lo que supone la revisión desde enfermería, de los procesos adaptativos que las personas presentan ante el cáncer, con miras a construir puentes seguros, donde los niños y adolescentes puedan transitar hacia una vida llena de segundas oportunidades.

\section{Objetivo}

Comprender los sentimientos de los niños y adolescentes con diagnóstico de cáncer a través de la indagación de los cambios que experimentan.

\section{Materiales y métodos}

La presente investigación es un estudio desarrollado bajo un enfoque cualitativo con aproximación fenomenológica. Para Sampieri et al., (21) este tipo de enfoques busca comprender un fenómeno donde los participantes construyen la realidad y le otorgan significados desde sus percepciones y representación, no se privilegia la utilización de datos numéricos. Dentro de este marco, se emplea el lenguaje natural para la recolección de los datos y la información obtenida estará influenciada por la experiencia de los participantes.

Apoyándonos en este concepto y en los postulados teóricos de Callista Roy, en la presente investigación se buscó comprender los sentimientos que tienen los niños y adolescentes con cáncer, al momento de ser diagnosticados con esta enfermedad y los cambios que se presentan a partir del diagnóstico, con miras a conocer el significado que le otorgan los sujetos del estudio a su realidad.

Los sujetos de estudio lo constituyeron niños y adolescentes con diagnóstico de cáncer entre 7 y 17 años de edad, atendidos en diferentes Instituciones prestadoras de servicios de salud en la ciudad de Montería, Córdoba (Colombia). El tipo de muestreo correspondió al no probabilístico y por bola de nieve, porque los participantes se escogieron a partir de las recomendaciones aportadas por otros sujetos de estudio que ya habían accedido a participar de la investigación. De modo similar, se fue contactando poco a poco a los demás participantes dando como resultado el rango de edad antes mencio- nado. Se optó por esta técnica de muestreo, porque es una población que presenta dificultades para su acceso y abordaje investigativo. Para la recolección de la información se empleó un instrumento con una primera sección de corte sociodemográfico para establecer: la edad del menor, nivel educativo, lugar de la entrevista, diagnóstico, entre otros. En segundo lugar se elaboraron diez preguntas que se formularon mediante una entrevista semiestructurada, donde se indagaba acerca de: qué sentiste cuando te informaron que estabas enfermo, cuánto tiempo ha pasado desde que recibiste el diagnóstico, piensas qué tu vida cambió, en qué cambio tu vida, cómo te sientes con esos cambios en tu vida, entre otras.

La muestra estuvo conformada por 5 participantes, dos fueron niños y tres adolescentes con quienes se obtuvo el nivel de saturación. Las entrevistas aplicadas a los niños y adolescentes fueron realizadas en presencia de sus cuidadores; en promedio cada entrevista duró 30 minutos. La técnica empleada permitió la expresión de los sentimientos y las respuestas fueron más abiertas; también se generó un ambiente de confianza. Las entrevistas fueron grabadas, con previa autorización consignada en consentimiento informado, para posteriormente ser transcritas. Las investigadoras colocaron seudónimos (nombres de héroes y dioses mitológicos) a cada participante, para garantizar el anonimato y realizar el análisis de contenido.

Además de la entrevista, se utilizó una guía de observación como mecanismo de recolección de la información. Antes y durante la entrevista se observaron aspectos tales como: expresiones corporales, condición física, imagen corporal, estado de ánimo, cambios físicos observables relacionados con el proceso natural de la enfermad y el tratamiento, vínculos afectivos, tono de voz, ritmo y velocidad, actitud, postura y gestos, entre otros. La observación fue realizada por la investigadora principal quien tiene experiencia en el campo de la investigación cualitativa y ha sido formada en este tipo de técnicas.

Para la interpretación de los datos se hizo uso del análisis de contenido de forma manual, teniendo en cuentas sus tres fases: análisis de contenido, análisis temático e interpretación. La triangulación de la información se realizó a partir del contraste entre los datos obtenidos en las entrevistas, la observación participante y la teoría empleada en el estudio. Los criterios éticos que rigen el presente estudio se enmarcan en los lineamientos de la Resolución 8430 de 1993, en la cual se establecen las normas científicas, técnicas y administrativas para la investigación en salud. 
La participación en la investigación de niños y adolescentes se realizó con previa autorización de los cuidadores a través del consentimiento informado. Para el control de sesgos se verificó previamente que los niños estuviesen en tratamiento oncológico y que fueran atendidos en la ciudad de Montería. Los datos recolectados fueron transcritos de forma textual, organizados mediante seudónimos, rotulados con códigos para invisibilizar el nombre real de los participantes y custodiados, garantizándose en todo momento la confidencialidad y el respeto por las expresiones de los participantes.

El estudio fue aprobado mediante Acta 5 de 18 de febrero de 2019, emitida por el Comité de Ética de la Facultad de Ciencias de la Salud de la Universidad de Córdoba.

\section{Resultados}

El total de participantes en la investigación correspondió a cinco, de los cuales dos eran niños y tres adolescentes. Las edades de los participantes oscilaron en un rango de edad de siete a diecisiete años; cuatro de sexo femenino y uno de sexo masculino. El nivel educativo varió entre primaria incompleta, bachillerato completo e incompleto; en la mayoría de los casos el cáncer más común fue la leucemia aguda (cuatro niños) y uno de meduloblastoma.

\section{Sentimientos inmersos en el diagnóstico de cáncer}

Esta categoría hace referencia a los sentimientos que experimentan los niños y adolescentes diagnosticados con cáncer, quienes están sujetos a las experiencias que viven durante el proceso de la enfermedad y son parte importante de la construcción del significado que le otorgan al diagnóstico del cáncer (Ver figura 1)

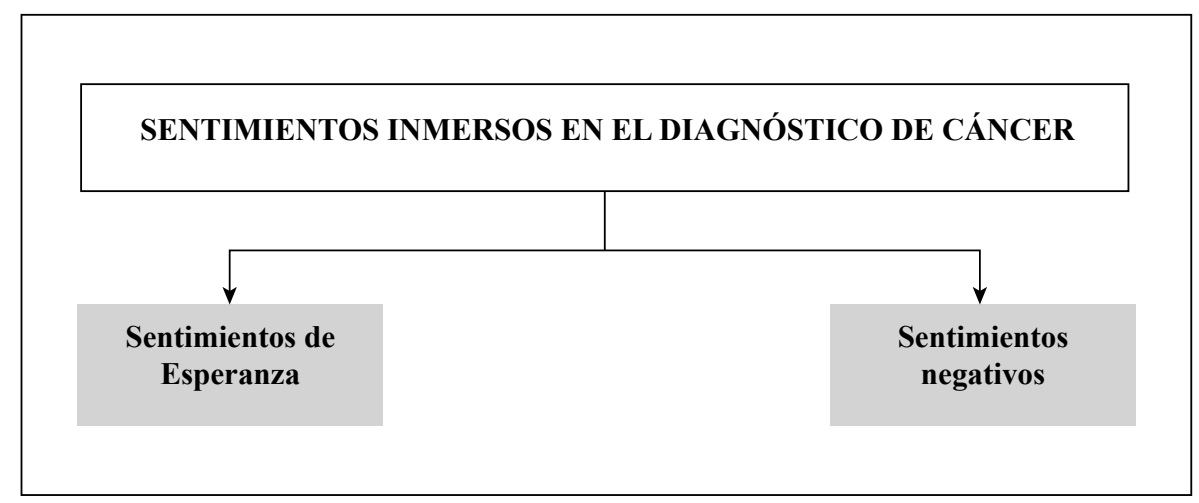

Figura 1. Sentimientos inmersos en el diagnóstico de cáncer

Fuente: Elaboración propia

El abordaje de esta categoría permitió develar dos subcategorías: la primera denominada sentimientos de esperanza, con la cual los niños expresaron las reacciones positivas que experimentaron a partir del diagnóstico del cáncer, con esperanza y optimismo. La segunda subcategoría, tiene que ver con los sentimientos negativos, donde los participantes expresaron temor a perder la vida y los conflictos se presentaron a partir del diagnóstico.

Los sentimientos que predominaron en los niños y adolescentes con cáncer fueron la esperanza y el optimismo; se aferraron a la vida para superar los retos y obstáculos que se presentaron en el proceso de su enfermedad; también, mostraban determinación para volver a realizar las actividades que solían hacer antes de ser diagnosticados con cáncer, como lo es asistir a la escuela, entre otros.
Así lo reflejan las siguientes expresiones:

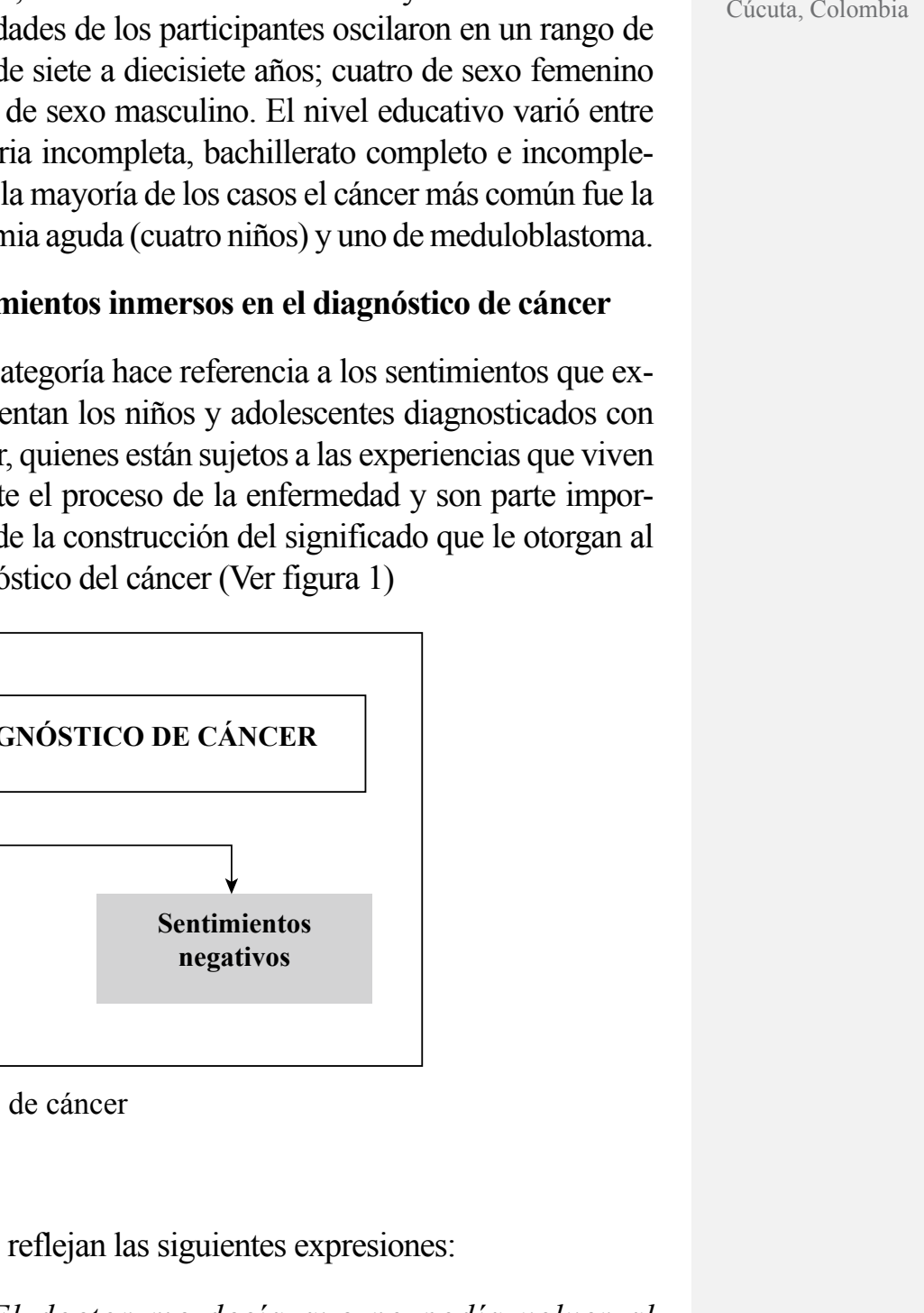

El doctor me decía que no podía volver al colegio, pero yo siempre le decía que sí, que yo quería (Atenea, la que posee profundos conocimientos).

El pensamiento siempre fue que me iba a salvar (Atenea, la que posee profundos conocimientos).

Con relación a los sentimientos negativos, las representaciones sociales del cáncer influyen en los sentimientos que tienen los niños sobre la enfermedad, particularmente con la creencia de que el cáncer es sinónimo de fallecimiento, lo que genera en ellos sentimientos negativos por el constante temor a fallecer. 
ISSN-PRINT

1794-9831

E-ISSN 2322-7028

Vol. 17 No. 2

May - Ago 2020

Cúcuta, Colombia
El miedo se apodera de los niños con cáncer y aflora en ellos sentimientos que expresan entre lágrimas e incertidumbre en relación con la representación de lo que significa el cáncer socialmente. Por otro lado, algunos niños son víctimas de bullying por parte de compañeros que desconocen la magnitud de la enfermedad; generalmente estas burlas hacen mención a los cambios físicos, tales como caída del cabello, adelgazar o presentar un semblante enfermizo.

Las anteriores afirmaciones son respaldadas por los siguientes testimonios:

Recuerda que yo te había dicho que tú sabes que el cáncer ha matado a muchas personas, bueno recuerda cuando yo te dije que yo no me quiero morir y que por eso y tú también te pusiste a llorar (Apolo, el que nunca muere)

Sentía mucho dolor, por la cosita que me metían (Apolo, el que nunca muere).
Bueno que te digo primero o sea yo no sabía en ese momento que era la leucemia ni nada de eso, solamente que un muchachito que fue bastante amigo mío el falleció por lo mismo y cuando a mi me dijeron eso, pues fue un poquito duro porque de la forma en la que me enteré, no fue la más conveniente, porque fue una muchachita la que se empezó a burlar de mí y ella me dijo tú tienes cáncer te vas a morir, tu familia no te quiere, se te va a caer el pelo y no sé qué (Helena, aquella que brilla o resplandece como una antorcha).

\section{Cambios en función del rol}

Esta categoría hace referencia a los cambios en la vida de los niños y adolescentes con cáncer en relación a su papel en la sociedad; en este sentido tienen cambios en el pensamiento y en las actividades y responsabilidades propias de su ciclo vital; es aquí donde se describen todos los acontecimientos que experimentan durante el proceso de la enfermedad (ver figura 2).

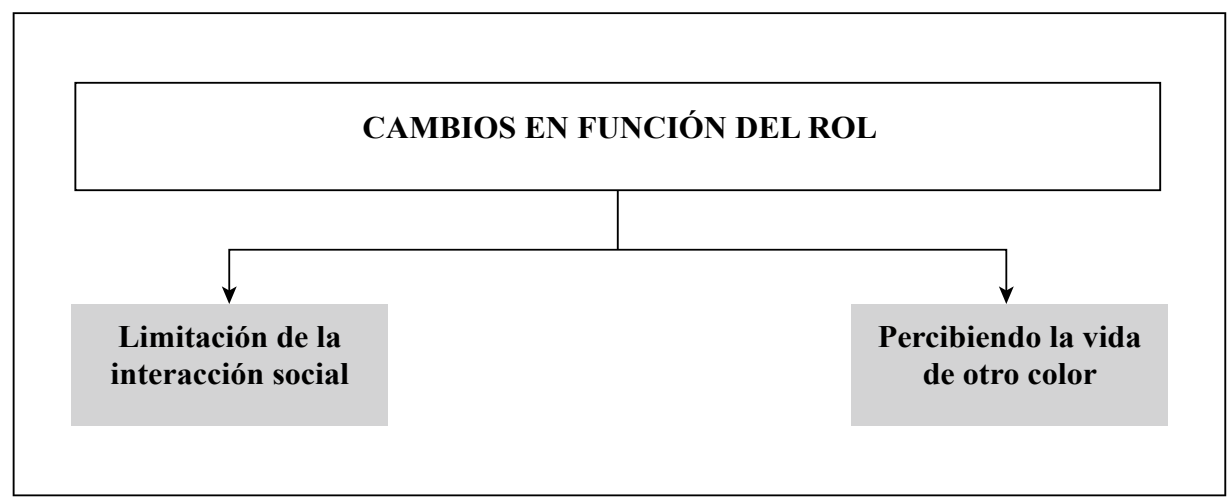

Figura 2. Cambios en función del rol Fuente: Elaboración propia

En la presente categoría surgieron dos subcategorías: la primera llamada limitación de la interacción social, hace referencia a los cambios sociales que experimentaron los niños y adolescentes como dejar de ir a la escuela, entre otros; la segunda denominada percibiendo la vida de otro color, en donde se describen los cambios a nivel personal que vivieron los participantes.

Con relación a la subcategoría limitación de la interacción social se pudo obtener lo siguiente:

El niño y adolescente con diagnóstico de cáncer experimenta de forma incomprensible la separación del ámbito escolar y el juego en las calles con sus amigos, para encontrarse con una nueva realidad, estar por largas tem- poradas en un centro médico y cambiar rotundamente la forma de cuidarse.

Así se evidenció en los siguientes testimonios:

Sí cambió, porque no pude ver a mis amigos y no pude ver a los seres queridos que amo, me sentía solo y triste todo el tiempo (Apolo, el que nunca muere)

Y ¿puedes salir a la calle? ¿Sí?, P. Un ratico, porque el doctor no me deja asolearme, quisiera ser como antes, cuando podía divertirme con mis amigos en la calle, ahora siempre estoy sola en mi casa, claro con mi mamá, pero yo quiero jugar con mis amiguitas (Niké, diosa de la victoria.) 
¿Y dejaste de asistir al colegio por la enfermedad?, P. Eee Si, si duré dos años sin estudiar, ahora que regresé, ya no tengo los mismos amigos, todos están más avanzados que yo, me siento aislada (Atenea, la que posee profundos conocimientos).

En las declaraciones inéditas de los participantes, se pudo evidenciar el desgate social que experimentan los niños y adolescentes con cáncer, generando sentimientos de tristeza y desolación, por no poder convivir socialmente como lo hacían antes de estar enfermos; son temas que los entristece y en ocasiones no quieren tratar. Además, la limitación social puede considerarse como una de las vivencias más difíciles de la enfermedad, al no poder disfrutar de la niñez y adolescencia plenamente, dejar la escuela, los juegos y salidas con los amigos. Como consecuencia, deben tratar de adaptarse a convivir con personal de la salud, los tratamientos médicos, equipos biomédicos, el olor particular de los hospitales, en un mundo de paredes y vestiduras blancas, sin los colores y las cosas por descubrir que ofrece el mundo fuera de los centros médicos.

Con relación a la subcategoría percibiendo la vida de otro color se puede evidenciar lo siguiente:

Vivir con cáncer significa para los niños y adolescentes un sin número de experiencias tanto negativas como positivas, que dejan una huella significativa en sus vidas y los hace percibir la vida de otro color.

La experiencia de tener cáncer para los niños y adolescentes que participaron de este estudio se ha convertido en una oportunidad, particularmente, porque tras el diagnóstico experimentaron un cambio a nivel personal, que los impulsó a transformar su forma de ver el mundo y a vivirlo. Los niños y adolescentes manifestaron que con el cáncer ellos aprendieron a apreciar cada segundo de la vida, mejorar las relaciones interpersonales, elegir mejor las amistades, entre otros.

Las expresiones así lo confirman:

Bueno antes no tenía tanta, así como esa comunicación con mis amigos y con mi familia pues tampoco, este, a partir de ahi conoci personas muy maravillosas, este le llegó esta casa mi mamá donde estamos aqui ahora y creo que ya, Aaaa se me han abierto muchas puertas a nivel académico y laborar en la clínica.
He cambiado mi forma de pensar, de ver la vida, porque antes veía la vida como que simple es la vida y me toca vivirla, pero ahora como que aprovecho más cada momento con mi familia, con mis amigos, con mi novio, porque él ha estado ahi en todo momento este y que veo la vida de otra manera de otro color (Respondió sonriendo).

Todo ha cambiado en lo fisico, en lo familiar y social, bueno como te digo, en la manera de pensar, porque antes únicamente, como te digo, vivía era pegada en el celular, era bastante grosera con mi familia (Helena, aquella que brilla o resplandece como una antorcha).

Esta experiencia a corta edad, contrario al imaginario que supone como un periodo de devastación, les sirve como una vivencia sobre nuevas oportunidades para crecer y convertirse en seres con una madurez que no es común para su edad; por este motivo, surgen cambios positivos en su entorno familiar, como lo son: expresión más abierta de sus sentimientos y mejor comportamiento, dejando a un lado las expresiones negativas hacia sus seres queridos, porque conciben que la familia se convierte en el todo para ellos.

\section{Discusión}

Los sentimientos de los niños y adolescentes sobre su diagnóstico de cáncer conjugan la interpretación que construyen ante los estímulos y la valoración consciente que realizan de los mismos. Atendiendo a las consideraciones anteriores, para la realización de la presente investigación se tomará como punto de análisis los sentimientos que se han derivado de los cambios fisiológicos, cognitivos y emotivos tras el diagnóstico de cáncer.

Desde la perspectiva de Roy, la adaptación es un concepto de gran valía en el marco de la relación enfermería, persona, salud y entorno, porque las personas se encuentran ante la búsqueda permanente de estímulos que puedan llegar a recibir del entorno, lo cual provoca una reacción que desencadena en la adaptación (22). A partir de esta afirmación teórica se derivan respuestas de adaptación favorables que promueven la integridad, permitiendo a las personas sobrevivir y controlar las situaciones adversas; en contraste, se hallan las respuestas de adaptación ineficaces que no admiten que se dé una adaptabilidad exitosa (23). 
Tras el diagnóstico de cáncer, los niños y adolescentes sufren una serie de cambios físicos que desde la perspectiva de Helena (aquella que brilla o resplandece como una antorcha) no se trata de algo en particular, sino que estos son percibidos como un todo al referirse a ellos como, "Todo ha cambiado en lo fisico"; es decir, que con el tratamiento farmacológico los niños y adolescentes logran tomar conciencia de los efectos secundarios de la enfermedad, los cuales se podrían identificar como: pérdida de cabello, náuseas, vómitos, cambios en la piel, diarrea y dolor ; así como, la conexión de estas alteraciones con los cambios sociales y psicológicos $(13,14,24)$.

Por su parte, Roy (22) agrega que la percepción es un proceso que enlaza el subsistema regulador con el relacionador, conectando los modos de adaptación, lo que podría traducirse en el hecho que los participantes dan cuenta de la conexión entre el modo de adaptación fisiológico y el auto concepto, al referirse a los cambios como una totalidad; es decir, que estos no solo les afecta su cuerpo, sino que también ven amenazada su vida social, psicológica y por ende la calidad de vida que limita las posibilidades de éxito de los tratamientos (25).

En tal sentido, las personas se consideran como sistemas holísticos de adaptación, cuyo entorno interno y externo, conformado por todos los fenómenos que envuelven el sistema de los seres humanos en el plano adaptativo, afectan el desarrollo de estas, así como las conductas que asumen ante diversos episodios de su vida. $\mathrm{Al}$ respecto, Roy (22) considera que el entorno es la principal fuente de estímulo de las personas que dependiendo de su carga emocional podrían amenazar o favorecer la existencia de un sujeto; de igual forma, el cuidado humanizado que ofrezca enfermería es clave para disminuir los sentimientos negativos que surgen durante el proceso (22). De ahí, que los lazos emocionales que construyen con las familias y los niños facilita la atención (23), de hecho, la enfermera se constituye en referencia para apoyar a niños, adolescentes y familiares para enfrentar las diferentes fases del cáncer, incluso la muerte (26).

Por tanto, desde la mirada de los participantes, vivir con cáncer se constituye en una experiencia traumática porque se relaciona con: los efectos secundarios de los tratamientos, los procedimientos médicos, el aislamiento social y la amenaza constante de muerte; resultados que coinciden con las apreciaciones de Fernández (27), quien enfatiza en la necesidad de tener en cuenta este tipo de alteraciones que afectan al niño y su familia.
Se debe precisar, que los niños y adolescentes con cáncer, experimentan cambios en función del rol y limitación de la interacción social, puesto que su nueva realidad los obliga a dejar de asistir a la escuela y alejarse de la mayoría de las personas que los rodean, por las estancias hospitalarias; por tanto, es un periodo que se caracteriza porque los juegos y alegrías se convierten en una etapa de cambios bruscos, que no hacen parte de su rol de niños y adolescentes en la sociedad.

Si bien, los cambios sociales y familiares son parte del proceso de ser un paciente oncológico, es de interés recordar que según el tipo de estímulos que se reciban se logrará una reacción eficaz o ineficaz en el marco de la adaptación a dicha enfermedad. Palacio y Zani (28) afirman que las representaciones sociales del cáncer están organizadas en torno al dolor, al sufrimiento y a la tristeza; así como también al cambio socio familiar, porque esta enfermedad se torna cruel y trae consigo pérdidas que desestabilizan el proyecto de vida por el aislamiento social, generando sufrimientos en el niño, por no recibir el apoyo social esperado de amigos $u$ otros profesionales de la salud (29).

Las expresiones de algunos de los participantes así lo confirman: "yo estudiaba en el colegio que nunca, o sea, nunca me dieron el apoyo, siempre yo iba a mi casa cada 26 días, duraba veinte días allá y me venía, pero de mi colegio nunca me fueron a visitar, siempre me dieron la espalda"; "no me senti nada bien porque mi mamá y mi papá terminaron y yo tenía una familia muy unida y desde que estoy enfermo ya no".

Sin embargo, los niños y adolescentes con diagnóstico de cáncer también perciben algunos sentimientos positivos que les permite adaptarse mejor a la enfermedad, especialmente cuando sienten que los cambios experimentados en todo el proceso se convirtieron en sí mismos en una oportunidad de crecimiento personal y una nueva forma de ver la vida; esta manera de percibir la enfermedad propicia la adaptación. Al respecto Roy (22) menciona que para lograr la adaptación, los individuos no tienen que liberarse de la enfermedad, más bien tienen que aprender a vivir con ella; es por eso, que los niños y adolescentes con cáncer no rechazan su realidad porque aprendieron a convivir con ella y a quedarse con lo mejor de esta enfermedad.

Vale la pena aclarar que estos sentimientos se evidencian más en los niños y adolescentes cuando hay mayor apoyo social y familiar, como se puede evidenciar en los 
siguientes comentarios: "pero bueno mi papá y mi mamá siempre me concientizaron de que tenía que ser fuerte y estar aferrada a Dios, y así sucesivamente, siempre tuve apoyo de mi familia y mis amigos, por eso no decai mucho"; "ahora como que aprovecho más cada momento con mi familia, con mis amigos, con mi novio porque él ha estado ahi en todo momento este y que veo la vida de otra manera de otro color"; "disfruto más la vida".

En definitiva, cuando el diagnóstico del cáncer infantil se asume en familia con el apoyo social idóneo, el proceso adaptativo se torna favorable (30), por tanto se inscribe en un antes y un después en sus vidas. A este respecto, es imprescindible precisar, que si bien las experiencias vividas durante el proceso de la enfermedad generan cambios importantes en sus proyectos de vida, la unión y la fuerza del grupo social provoca emociones positivas y optimistas; igualmente, fortalecen las estrategias para enfrentar los momentos difíciles y los obstáculos que se presentan en el duro camino de la enfermedad, convirtiéndose en un dispositivo que cohesiona la familia y su entorno inmediato (31).

\section{Conclusiones}

- Los niños y adolescentes con cáncer reflejan sentimientos de esperanza y optimismo que los ayudan a superar las duras pruebas de la enfermedad, se convierten en personas más fuertes que creen en sí mismos y en sus capacidades. A la luz de la teoría de Sor Callista Roy, estos niños y adolescentes con diagnóstico de cáncer, muestran características que podrían considerarse fundamentales para el modo de adaptación y de auto concepto, porque reflejan en sí mismos la autoconfianza y la esperanza para sobreponerse a la enfermedad.

- A pesar que los adolescentes y especialmente los niños, son seres que aún no alcanzan a conocer o entender en su totalidad la dinámica del mundo, en el caso del cáncer no desconocen la magnitud de esta enfermedad y sus repercusiones, es por ello que experimentan miedos, dolor, preocupación, temor a la muerte e incertidumbre y no son ajenos a su nueva realidad. Además, deben enfrentarse a las representaciones sociales del cáncer, convivir con los cambios en la dinámica familiar propios de cualquier familia y los atribuidos a las demandas de la enfermedad.

- Los niños y adolescentes con cáncer se ven obligados a limitar su interacción social debido a las características de la enfermedad, exigiéndoles que abandonen ciertas actividades que solían hacer antes de ser diagnosticados, como lo son: asistir a la escuela, interactuar con los amigos y cambios en la dinámica familiar; todo lo anterior es causado por las largas estancias hospitalarias, que los alejan de los familiares y amigos más cercanos; sin embargo, a pesar de todas estas limitaciones persisten en el deseo de superación y en las ganas de vivir.

- En definitiva, los niños y adolescentes con cáncer le otorgan a esta experiencia un significado de cambio y crecimiento personal; es por eso, que la forma de vivir la vida y de percibir el mundo y las nuevas vivencias a las cuales se enfrentan los convierten en seres humanos optimistas, capaces de superar las adversidades, tomando lo bueno que les dejó cada experiencia vivida durante la enfermedad. Por esta razón, le otorgan un nuevo sentido a sus vidas, aprovechando los gestos y momento de amor que viven con la familia y seres queridos; esto es, que hacen a un lado los estigmas de un mundo que en ocasiones se torna de matices grises, para darle cabida a un mundo de mil colores, donde abunda la fe, el optimismo, los deseos de superar las adversidades y el amor por los demás.

\section{Conflicto de intereses}

Las autoras declaran no tener ningún conflicto de intereses.

\section{Referencias bibliográficas}

1. Organización Mundial de la Salud. Cáncer. Datos y cifras. 2018 [Internet]. [citado el 30 de julio de 2019]. Disponible en: http://www.who.int/es/news-room/fact-sheets/detail/cancer

2. Ministerio de Salud y Protección Social. Boletín de Prensa No 020 de 2018. [Internet]. Bogotá; 2018. [citado el 30 de julio de 2019]. Disponible en: https://www.minsalud.gov.co/Paginas/Salud-y-vidapara-los-ni $\% \mathrm{C} 3 \% \mathrm{~B} 1$ os-con-cancer.aspx 
3. Instituto Nacional de Salud. Boletín Epidemiológico Semanal. [Internet]. Bogotá; 2019 [consultado 14 de abril 2020]. Disponible en: https://www.ins.gov.co/buscador-eventos/BoletinEpidemiologico/2019_Boletin_epidemiologico_semana_52.pdf

4. Bravo L, Muñoz N. Epidemiología del cáncer en Colombia. Colomb Med. [Internet]. 2018 [citado el 30 de julio de 2019]; 49(1):09-12. Disponible en http://www.scielo.org.co/pdf/cm/v49n1/es 16579534-cm-49-01-00009.pdf

5. Arrutia C. Proceso de terminalidad y muerte: experiencias y significados en usuarios (as) con cáncer, sus familiares y el equipo de salud en el modelo de cuidados paliativos de Clínica Familia [Tesis en Internet], Julio de 2015 [consultado 25 de abril 2019]. Santiago de Chile: Universidad de Chile. Disponible en: http://repositorio.uchile.cl/bitstream/handle/2250/140305/Memoria\%20Carla\%20Urrutia\%20FINAL.pdf?sequence $=1 \&$ isAllowed $=y$

6. Sánchez-Egea R. El tratamiento psicológico en familiares de niños con cáncer: Un estudio metaanalítico [tesis doctoral]. España: Universidad de Murcia; 2017. 569 p.

7. Fonseca-Salguero MA, Rojas-Vargas JC, Cubillos-Moreno PA, Moreno-Ozuna LI, Carreño-Moreno SP. Concepto de sobrecarga del cuidador del niño con cáncer: revisión integrativa. Rev. cienc. cuidad. [Internet]. 2019 [citado el 30 de julio de 2019]; 16(2):120-131. Disponible en https://revistas.ufps.edu. co/index.php/cienciaycuidado/article/view/1605/1508

8. Fajardo-Rocha H, Montalvo-Prieto A. Competencias del cuidador familiar para cuidar a su familiar que recibe quimioterapia. Rev. Cienc. cuidad. [Internet]. 2019 [citado el 30 de julio de 2019]; 16(2): 95-107. Disponible en https://revistas.ufps.edu.co/index.php/cienciaycuidado/article/view/1612/1506

9. Montgomery K, Sawin KJ, Hendricks-Ferguson VL. Experiences of pediatric oncology patients and their parents at end of life: a systematic review. J Pediatric Oncol Nurs. [Internet]. 2015 [citado el 30 de julio de 2019]; 33(2):85-104. Available from: http://citeseerx.ist.psu.edu/viewdoc/download?doi=1 0.1.1.1010.7838\&rep=rep1\&type $=$ pdf

10. Ramírez-Perdomo CA, Rodríguez-Vélez ME, Perdomo-Romero AY. Uncertainty in the face of the cancer diagnosis. Texto Contexto Enferm. [Internet]. 2018 [citado el 23 de agosto de 2019]; 27(4): 2-9. Disponible en http://www.scielo.br/pdf/tce/v27n4/en_0104-0707-tce-27-04-e5040017.pdf

11. E Sousa AD, Resende Da Silva LF, Paiva ED. Nursing interventions in palliative care in Pediatric Oncology: an integrative review. Rev Bras Enferm [Internet]. 2019; 72(2):531-540. Available from: http://www.scielo.br/scielo.php?script=sci_arttext\&pid=S0034-71672019000200531

12. Bellver A, Verdet H. Adolescencia y cáncer. Psicooncología. [Internet]. 2015 [citado el 30 de julio de 2019]; 12(1):141-156. Disponible en: http://revistas.ucm.es/index.php/PSIC/article/viewFile/48909/45635

13. Muñiz D, Jaime R, Ojeada A. Alternativa psicopedagógica para potenciar la resiliencia en pacientes oncopediátricos. Maestro y Sociedad. [Internet]. 2019 [citado el 23 de agosto de 2019]; 16(1):82-96. Disponible en: https://revistas.uo.edu.cu/index.php/MyS/article/view/4817/4185

14. García-García A, Lucio Gómez-Maqueo, M. Estilo de afrontamiento y calidad de vida en adolescentes con cáncer. Gaceta Mexicana de Oncología. [Internet]. 2016 [citado el 23 de agosto de 2019]; 15(1): 3-9. Disponible en: https://www.sciencedirect.com/science/article/pii/S1665920116000079

15. Cely-Aranda JC, Duque-Aristizabal CP, Capafons A. Psicooncología pediátrica. Diversitas: Perspectivas en Psicología. [Internet]. 2013 [citado el 23 de agosto de 2019]; 9(2):289-304. Disponible en: http:// www.scielo.org.co/scielo.php?script $=$ sci arttext\&pid $=$ S1794-99982013000200005\&lng=en\&tlng=es

16. Pozo Muñoz C, Bretone Nieto B, Martos Méndez MJ, Morillejo EA, Cid Carrique N. Repercusiones psicosociales del cáncer infantil: apoyo social y salud en familias afectadas. Revista Latinoamericana de psicología [Internet]. 2015 [citado el 23 de agosto de 2019]; 97(2), 93-101. Disponible en: https:// www.sciencedirect.com/science/article/pii/S0120053415000047

17. Llantá-Abreu M, Grau-Ábalo J, Bayarre-Vea HD, Renó-Céspedes J, Machín-García S, Verdecia- 
Cañizares C. Calidad de vida relacionada con la salud en niños y adolescentes con cáncer atendidos en servicios de Oncohematología de La Habana, 2011-2013. Rev haban cienc méd. [Internet]. 2016 Abr [consultado 15 de julio de 2019]; 15(2):85-296. Disponible en: http://scielo.sld.cu/scielo. php?script $=$ sci arttext\&pid $=$ S1729-519X2016000200016\&lng $=$ es

18. Gonzales R, Madero, L. El cáncer en el mundo. Rev Esp Pediatr. [Internet]. 2015 [consultado 10 de febrero 2019]; 71(1): 31-38. Disponible en: http://www.seinap.es/wp-content/uploads/Revista-dePediatria/2015/REP\%2071-1.pdf\#page=31

19. Cárdenas F, Gómez O. Análisis de situación de enfermería: cuidando la familia desde el modelo de adaptación de Roy. [Internet]. 2018 [consultado 10 de febrero 2019]; 10(1), 93-101. Disponible en internet: https://revistacuidarte.udes.edu.co/index.php/cuidarte/article/view/601/1068

20. Bonilla Cruz N, Alarcón Carvajal M, Sánchez Meza G. Familia: un estado del arte en grupos de investigación de Colciencias en psicología entre 2010-2015. Mundo FESC, [Internet]. 2016; 6(11),33-47. Disponible en: https://www.fesc.edu.co/Revistas/OJS/index.php/mundofesc/article/view/80/129

21. Sampieri R, Fernández C, Baptista P. Metodología de la investigación. 3 ed. México: McGraw-Hill Interamericana, 2004. 10-14 p.

22. Tomey A, Alligood M. Modelos y teorías en enfermería, $7^{\mathrm{a}}$ Ed, Barcelona, España: Elsevier, 2008. 342-354 p.

23. Campiño-Valderrama SM, Duque PA. Afrontamiento y adaptación de cuidadores de niños y niñas con cáncer. [Internet]. Rev Univ. Salud. 2016 [consultado 10 de febrero 2019]; 18(2):302-311. Disponible en http://www.scielo.org.co/pdf/reus/v18n2/v18n2a11.pdf

24. Cadena-Villa A, Castro Retamal G, Franklin de Martínez H. Vivencias y significados de adolescentes sobrevivientes al cáncer. Trilogía. Ciencia y Tecnología y sociedad. 2014; 10 (1): 145-163.

25. Grau C, Espada MC. The perceptions of parents of children with cancer about changes in Family relations. Psicooncología [Internet]. 2012 [consultado 10 de febrero 2019]; 9(1):125-136. Available from https://revistas.ucm.es/index.php/PSIC/article/view/39142

26. Da Rosa Reis T, Cardozo de Paula C, Potrich T, De Mello Padoin SM, Bin A, Flores Mutis C, De Moura Bubadué $\mathrm{R}$. Relações estabelecidas pelos profissionais de enfermagem no cuidado as crianças com doença oncológica avançada. Aquichan [Internet]. 2014[consultado 10 de febrero 2019]; 14(4):496508. Available from: http://www.scielo.org.co/pdf/aqui/v14n4/v14n4a05.pdf

27. Fernández M. El cáncer en la infancia y adolescencia: consecuencias en el paciente, la familia y papel del asociacionismo. Facultad de psicología y educación. Trabajo fin de grado de pedagogía [Internet], 2015 [consultado 10 de febrero 2019]. Disponible en: http://dadun.unav.edu/handle/10171/39669

28. Palacio J, Zani B. Representaciones sociales del cáncer y de la quimioterapia. Revista Diversitas Perspectivas en Psicología. [Internet]. 2014 [consultado 24 de junio del 2019]. Disponible en: http:// www.scielo.org.co/pdf/dpp/v10n2/v10n2a03.pdf

29. Amorim ME, Moura de Moura F, Marinho-Albuquerque T, Altamira-Pereira da Silva R, Collet N. Network and social support in children with chronic diseases: understanding the child's perception. Texto Contexto Enferm. [Internet]. 2017 [consultado 24 de junio del 2019]; 26(1): 2-10. Disponible en http://www.scielo.br/pdf/tce/v26n1/0104-0707-tce-26-01-e6980015.pdf

30. Valencia-Jiménez, NN, Ortega-Montes JE, Puello-Alcocer EC. Hospital pedagogy: A space of love and recognition for the oncological pediatric patient. [Internet]. 2019 [consultado 24 de junio del 2019]; 28(4):1-14. Available from http://www.scielo.br/pdf/tce/v28/1980-265X-tce-28-e20180112.pdf

31. Melguizo-Garín A, Martos-Méndez MJ, Hombrados-Mendieta, I. Influencia del apoyo social sobre el estrés y la satisfacción vital en niños con cáncer desde una perspectiva multidimensional. Psicooncología. [Internet]. 2019 [consultado 10 de febrero 2019]; 16(1): 25-42. Disponible en: https://search. proquest.com/openview/4c9299623f091bac5f23054091725dbd/1?pq-origsite $=$ gscholar \&cbl=54850 